\title{
Risques et bénéfices de la transgenèse vis-à-vis de l'allergie
}

Oléagineux, Corps Gras, Lipides. Volume 9, Numéro 2, 107-11, Mars - Juin 2002, La filière

Auteur(s) : Denise-Anne MONERET-VAUTRIN, Université Poincaré Nancy I, Service de médecine interne, Immunologie clinique et allergologie, Hôpital central, 29, avenue de Lattre-de-Tassigny, 54035 Nancy.

Résumé : L'industrialisation de l'alimentation comporte de nombreux récents développements, appliqués à toutes les étapes, des matières premières aux produits alimentaires terminaux [1]. Les techniques agronomiques appliquées à la sélection d'espèces et de variétés, à leur hybridation, à la commercialisation d'aliments nouveaux, n'ont pas, jusqu'ici, attiré de craintes du public. En revanche, la commercialisation d'aliments obtenus à partir d'organismes génétiquement modifiés a provoqué de sensibles mouvements d'opinion, attribuant des risques particuliers à ces OGM. Parmi ces risques, le risque allergique a été allégué. Le passage intempestif dans l'alimentation américaine d'un maïs transgénique destiné à l'alimentation du bétail a été suivi de la déclaration de dizaines de cas " d'allergie " à un maïs génétiquement modifié, contenant la protéine cry9 (provenant de Bacillus thuriengensis, un insecticide), et a motivé le retrait de commercialisation de ce maïs aux États-Unis [2]... Si cet épisode caractérise très probablement un fantasme, il n'en reste pas moins vrai, d'une part que le risque allergique d'un aliment transgénique a été démontré [3] et que, d'autre part, les allergies alimentaires sont fréquentes dans tous les pays développés, avec une moyenne générale de 3 à $4 \%$, et de $8 \%$ en population pédiatrique [4]. Un certain nombre alarme par la gravité, ou fait obstacle à une vie normale par la chronicité. Dans la mesure où les OGM seront une part nécessaire de l'alimentation du futur proche, il est légitime d'examiner les précautions nécessaires pour réduire le risque allergénique, avant de s'intéresser aux bénéfices attendus des OGM dans la prévention ou le traitement d'affections allergiques.

\section{ARTICLE}

Excepté dans le domaine de la recherche fondamentale, souvent développée à la fois par des organismes publics bénéficiant d'aides gouvernementales et par quelques multinationales, le financement de la recherche provient essentiellement des retours financiers procurés par la mise sur le marché des brevets et des produits qui en sont issus. Afin de garantir un amortissement des frais et de permettre des retours sur les lourds investissements nécessaires, des instruments juridiques portant sur la propriété intellectuelle ont été créés. Dans le domaine des biotechnologies et de l'amélioration des plantes, il a été prévu des dispositifs accordant aux détenteurs de droits, d'exploiter le résultat de leurs recherches, seuls ou sous leur contrôle, par l'intermédiaire de licences d'exploitation.

\section{Protection de l'obtention végétale}

Compte tenu de l'impossibilité de décrire les procédés d'obtention et de sélection et leur résultat par brevet, I'Union internationale pour la protection des obtentions végétales (Upov) a élaboré en 1961 une convention permettant de protéger les nouvelles variétés végétales sur la base de trois critères phénotypiques : 
- la distinction de la variété, qui doit être nouvelle par rapport à celles qui sont notablement connues ;

- I'homogénéité de la variété qui doit être composée de plantes identiques ;

- la stabilité de la variété aux différents stades successifs de la production.

Jusqu'au développement des biotechnologies, les obtenteurs ne possédaient que ce seul moyen pour protéger leurs nouvelles variétés, tout en permettant à tous d'utiliser le génome de l'espèce à partir de matériel génétique protégé ou non, comme source initiale de variation, pour la sélection et l'amélioration de variétés nouvelles.

\section{Protection des inventions biotechnologiques}

L'évolution des sciences du vivant, qui tend à modifier les caractéristiques du génome commun à une plante ou à une espèce, permet aujourd'hui de décrire les méthodes et les procédés scientifiques, de définir le caractère inventif de ces méthodes et procédés et caractérise l'intérêt industriel ou économique de ces procédés ou des produits qui en sont issus, tous critères qui sont nécessaires à la brevetabilité. En conséquence, en Europe, une directive communautaire a prévu une protection juridique spécifique des inventions biotechnologiques, qui s'intègre, pour chaque État membre, dans le cadre des dispositions des protections par brevet. Cette directive exclut néanmoins la brevetabilité des procédés essentiellement biologiques et limite la protection lorsque les recherches et leurs résultats portent sur l'être humain. Elle exclut donc de facto la protection des variétés par brevet, mais permet d'y intégrer des procédés ou matériels brevetés.

Dans beaucoup d'autres pays hors de l'Union européenne, et en particulier en Amérique du Nord, il est non seulement permis de breveter les inventions biotechnologiques et leurs résultats, mais également et par voie de conséquence, les variétés qui les portent. Aux États-Unis, certaines variétés essentiellement biologiques ressortent, pour leur protection, de la législation sur les brevets.

\section{Les enjeux}

Les enjeux de la recherche et de l'amélioration végétale, au même titre que le développement de plus en plus rapide de la génomique et des biotechnologies, rendent nécessaire une réflexion sur les deux systèmes de protection existants et sur la compétition, pour ne pas dire la concurrence, qui existera à l'avenir entre les métiers en amont de la génomique et des biotechnologies, et les métiers en aval que sont la sélection et l'amélioration des plantes. Les deux peuvent d'ailleurs être liés, mais nécessitent une industrie très importante et un chiffre d'affaires considérable, permettant des investissements très importants à long terme avant de bénéficier de résultats économiques et commerciaux.

La disparité des moyens de protection risque de créer des différences entre les industries de l'Union européenne et celles de nombreux pays développés. Si l'on ajoute à cette différence juridique une différence d'appréciation dans l'utilisation des organismes génétiquement modifiés, d'importantes questions se posent sur l'avenir communautaire des recherches et sur la compétitivité des produits que les producteurs de l'Union européenne pourraient mettre sur le marché à moyen et à long terme. 


\section{CONCLUSION}

\section{L'avenir}

La plupart des sélectionneurs, en règle générale des entreprises moyennes, sont attachés au fait de pouvoir librement utiliser le génome d'une plante pour améliorer les variétés appelées à être mises sur le marché. Ils sont donc partisans de l'utilisation de variétés, protégées ou non, afin de les améliorer. La protection des plantes ne protège pas le génome, mais uniquement le travail d'amélioration du sélectionneur. L'amélioration des plantes permet un progrès continu des variétés par une libre concurrence des travaux de recherche et de leurs résultats.

Dans le cadre de la brevetabilité, les conditions d'utilisation du brevet sont totalement différentes. Le brevet ne protège pas par différence, comme le certificat d'obtention végétale, mais par ressemblance. Un brevet est donc extrêmement large dans ses revendications et peut permettre, pendant la durée de protection, d'empêcher toute expérimentation et utilisation du procédé et du résultat du procédé par des tiers, ce qui, outre des problèmes de concurrence lorsque le brevet est majeur, crée un statut de non-concurrence au brevet et à la variété qui en est issue, pendant une période assez longue pour décourager toute recherche concurrente.

La différenciation entre l'application de la protection en Europe et en Amérique du Nord risque donc de poser de nombreux problèmes à la fois scientifiques et juridiques, puisque les conditions d'utilisation du matériel génétique ne seront pas identiques et que le droit des brevets possède une force que ne possède pas le certificat d'obtention végétale dans le domaine de l'exclusivité et de la fermeture à toute expérimentation par des tiers du matériel génétique breveté.

Les industries utilisatrices de productions agricoles de grandes cultures peuvent donc légitimement s'inquiéter de la compétitivité des productions européennes au même titre que de la perennité de la recherche communautaire. Si l'on ajoute à ce phénomène, la différence de comportement vis-à-vis des sciences de la vie, des biotechnologies et des organismes génétiquement modifiés, par les scientifiques, les politiques et les sociétés civiles entre l'Europe et le reste du monde, on peut craindre une future dépendance des productions par rapport aux firmes pouvant librement développer les sciences de la vie et celles qui ne peuvent, réglementairement et socialement, les expérimenter et mettre leurs résultats en production en Europe.

La conséquence pourrait être une uniformisation des productions ou des sources d'approvisionnement importées, avec comme corollaire, une restriction des offres du marché, une diminution de la concurrence et des différentes qualités ainsi qu'une dépendance des industries européennes par leur approvisionnement. 


\section{Illustrations}

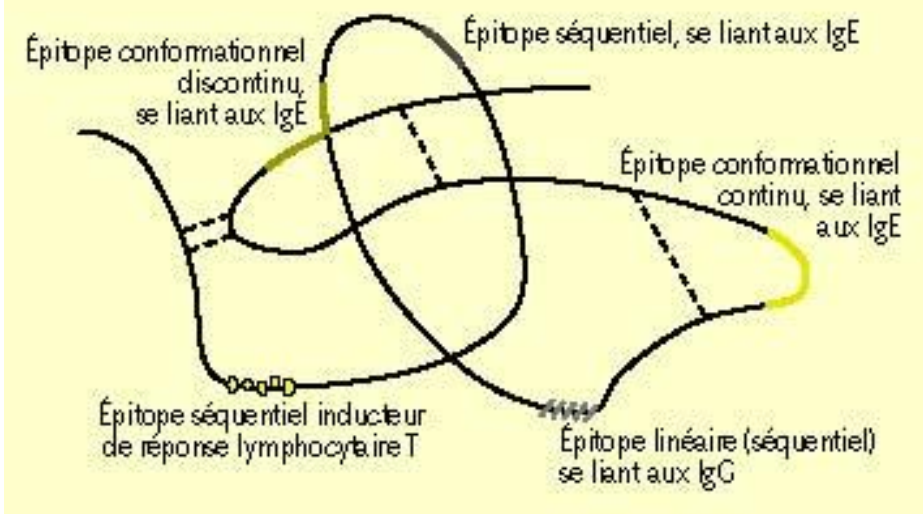

Figure 1. Schéma d'un allergène.

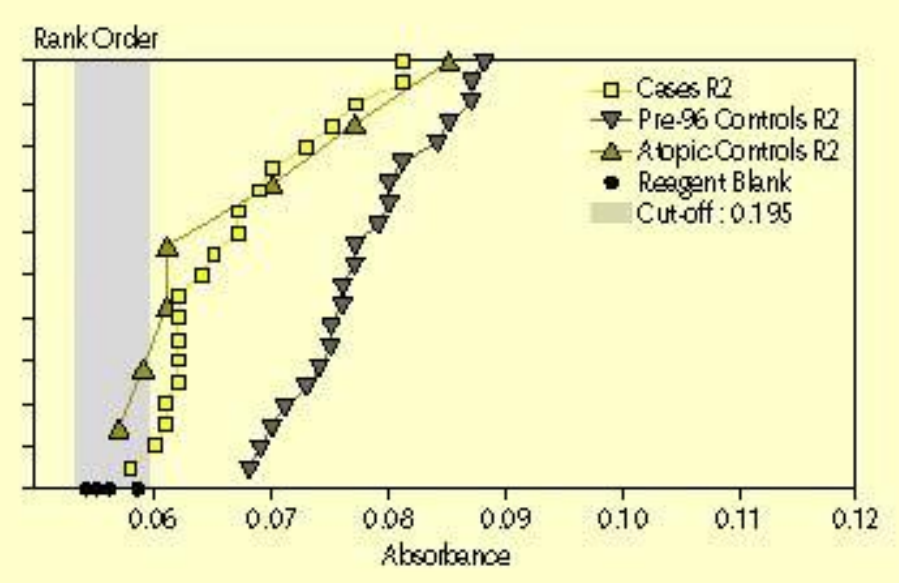

Figure 2. Exemple de la protéine Cry9c (maïs OGM). 